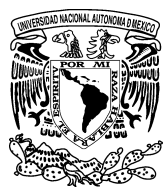

\title{
¿Influyen las Normas Internacionales de Información Financiera en el riesgo de las acciones?
}

\author{
Influencing the International Financial Reporting \\ Standard in the equity risk?
Gokhan Boz $^{\mathrm{a}}$, Carlota Menéndez Plans ${ }^{\mathrm{a}, *}$, Neus Orgaz Guerrero ${ }^{\mathrm{b}}$ y Diego Prior Jiménez ${ }^{\mathrm{a}}$
a Universidad Autónoma de Barcelona, España
b Universidad Oberta de Catalunya, España

Recibido el 31 de enero de 2014; aceptado el 8 de octubre de 2014

Disponible en Internet el 27 de junio de 2015

\begin{abstract}
Resumen
El objetivo del presente trabajo de investigación es doble: estudiar qué información explica el riesgo de las acciones y analizar si la implantación de las Normas Internacionales de Información Financiera (NIIF), realizada en el mercado español en 2005, influye en dicha información. Conocer qué información contable y/o macroeconómica explica el riesgo de una acción es una información útil para el empresario y para el profesional de la gestión, pues nos indica qué variables observar para estimar la evolución del riesgo y, en consecuencia, la evolución del coste de capital o tasa de actualización. La tasa de actualización es una variable muy importante en muchas decisiones financieras, y su valor, que depende entre otras variables del riesgo, tiene una gran influencia en la decisión. Por este motivo es importante que dicha tasa se determine de forma objetiva, algo especialmente difícil en las pequeñas y medianas empresas (PYMES). Una gestión eficiente implica poder anticiparse a los efectos futuros, actuar para capturar los efectos positivos y minimizar los efectos negativos. Si sabemos qué variables explican el riesgo, conoceremos qué indicadores observar para analizar su comportamiento y prever lo que vaya a suceder en el futuro sobre la tasa de actualización. Derechos Reservados (C 2015 Universidad Nacional Autónoma de México, Facultad de Contaduría y Administración. Este es un artículo de acceso abierto distribuido bajo los términos de la Licencia Creative Commons CC BY-NC-ND 4.0.
\end{abstract}

Palabras clave: Riesgo; Normas Internacionales de Información Financiera (NIIF); Pequeñas y medianas empresas (PYMES); Coste de capital de las acciones; Información contable; Macroeconómica

\footnotetext{
* Autor para correspondencia.

Correo electrónico: Carlota.Menendez@uab.cat (C. Menéndez Plans).

La revisión por pares es responsabilidad de la Universidad Nacional Autónoma de México.
} 


\begin{abstract}
The present research has two objectives. First, we study the determinants of stock risk. Second, we analyze whether International Financial Reporting Standard (IFRS) implemented in the Spanish market in 2005 has affected these determinants. It is quite important for both entrepreneurs and management professionals to understand the accounting information and macroeconomic factors that explain stock risk, since it suggests which factors can be used to estimate this risk, and hence, to analyze the evolution of cost of capital or discount rate. The discount rate plays an important role in wide range of financial decisions; whose value depends on the risk among other factors. Therefore, it is significant to obtain an objective estimation of discount rate, which is difficult to handle specifically in the context of small and medium sized enterprises (SMEs). Effective management involves the ability to forecast future changes, capture positive effects, and minimize negative ones. Once we find out the variables that can be utilized to explain the risk, we can observe and analyze their evolution to anticipate future changes in the discount rate.

All Rights Reserved (c) 2015 Universidad Nacional Autónoma de México, Facultad de Contaduría y Administración. This is an open access item distributed under the Creative Commons CC License BY-NC-ND 4.0.

Keywords: Risk; International Financial Reporting Standard (IFRS); Small and medium enterprises (SMEs); Cost of equity capital; Accounting information; Macroeconomic information
\end{abstract}

\title{
Introducción
}

Son muchas las decisiones empresariales que necesitan disponer de una medida objetiva del riesgo, por ejemplo, analizar si una nueva inversión puede crear valor para el accionista o calcular el valor de mercado de un negocio que deseamos comprar o vender. Conocer el riesgo nos permite determinar la prima de riesgo y, consecuentemente, el coste de capital o tasa de actualización ${ }^{1}$ de los futuros flujos libres de tesorería, cuyo valor tiene una gran influencia en cualquier proceso de valoración, pues reduciendo (aumentando) la tasa de actualización aumenta (disminuye) el valor de mercado del activo que se valora. Por este motivo, es importante que esta tasa de actualización se fije de forma fiable y objetiva.

Para las empresas cuyas acciones cotizan en bolsa existen métodos. como por ejemplo el Capital Asset Pricing Model ${ }^{2}$ (CAPM), para estimar el riesgo de las acciones de forma objetiva ${ }^{3}$. El CAPM mide el riesgo calculando la variabilidad del precio de las acciones respecto a un índice bursátil de referencia. A esta medida del riesgo se la denomina comúnmente «beta de mercado». Para las empresas no cotizadas, las cuales representan más del $80 \%$ de la estructura empresarial española, el riesgo de mercado de las acciones (la beta de mercado) no se puede estimar porque

\footnotetext{
${ }^{1}$ La tasa de actualización o rentabilidad mínima exigida es siempre el resultado de sumar al tipo de interés libre de riesgo una prima por el riesgo. El principal problema en esta estimación se encuentra, para las PYME, en la estimación objetiva de la prima de riesgo.

2 Aunque existen otros métodos para estimar el riesgo de las acciones, el CAPM es, a pesar de sus limitaciones, un método vigente que proporciona buenos resultados, tal y como ponen de manifiesto los libros de texto más profusamente utilizados en las mejores escuelas de negocios del mundo. Véase, por ejemplo, Brealey, Myers y Allen, 2010; Damodaran (2012).

3 Objetiva en la medida de lo posible, ya que una objetividad del $100 \%$ es imposible de conseguir, pues aunque el mercado de capitales sea eficiente, los movimientos en los precios de las acciones vienen determinados por las decisiones que toman los inversores. Es objetiva en el sentido de que no está influenciada por los intereses particulares del momento.
} 
desconocemos la serie temporal de los precios de las acciones. En consecuencia, si no encontramos la forma de paliar esta limitación, es posible que tomemos decisiones sobre estas empresas usando una información incompleta, poco precisa y, en algunas ocasiones, subjetiva.

Una posible vía de solución a este problema se inició con Beaver, Kettler y Scholes (1970). Este trabajo busca qué variables contables explican el riesgo de mercado de las acciones de empresas cotizadas norteamericanas. A partir de este artículo seminal se desarrollan múltiples trabajos analizando la conexión entre la información revelada por las empresas y los movimientos de los mercados de capitales. Sin embargo, la inmensa mayoría de ellos continúa utilizando datos norteamericanos y sus resultados distan de ser concluyentes. Teniendo en cuenta que el riesgo de mercado no es el mismo en todas las economías, Foster, Kasznik y Sidhu (2012) muestran la importancia del factor país en el poder explicativo de los modelos, y parece lógico preguntarse qué relación hay en el mercado español entre la información contable y macroeconómica y el riesgo de las acciones.

Con este artículo queremos contribuir a la solución del problema anterior estudiando una muestra de empresas españolas con un doble propósito: 1) estudiar si existe una relación significativa y estable en el tiempo entre la información contable y macroeconómica y el riesgo de mercado de las acciones (o riesgo sistemático), y 2) comprobar si la aplicación de las Normas Internacionales de Información Financiera (NIIF) influye en la conexión entre la información y el riesgo sistemático de las acciones.

Conocer qué información explica el riesgo de las acciones es útil para el empresario, y también para el analista financiero, pues identifica qué características de la empresa y de la economía influyen en el riesgo de sus acciones. Esta información es una pauta que señala las variables a tener en cuenta para medir el riesgo de las PYME de una manera más objetiva, para posteriormente establecer la rentabilidad mínima exigida o coste de capital. Estudiar el impacto de las NIIF es útil, pues permite valorar si su implantación mejora el nivel de calidad de la información divulgada o si cambia la información que explica el riesgo.

El desarrollo de la investigación empírica nos permite confirmar que la información que explica el riesgo es una combinación de información contable y macroeconómica y que la información que explica el riesgo difiere para las empresas financieras e industriales.

El artículo sigue el siguiente orden. A partir de esta introducción encontramos una revisión de la literatura existente, posteriormente el diseño de la investigación y a continuación, en el cuarto apartado, comentamos los resultados más significativos de las diferentes estimaciones realizadas y, para finalizar, encontramos las conclusiones e implicaciones más importantes para la gestión empresarial de los resultados obtenidos.

\section{Revisión de literatura}

El punto de partida de la investigación que aquí se desarrollada se encuentra en el trabajo de Beaver et al. (1970), considerado pionero en la búsqueda de una relación entre el riesgo sistemático de las acciones y la información contable de la empresa. Posteriormente aparecieron múltiples trabajos con el mismo fin: Breen y Lerner (1973), Lev y Kunitzky (1974), Lev (1974), Bildersee (1975), Agusman, Monroe, Gasbarro y Zumwalt (2008), Menéndez, Prior y Orgaz (2012) y Papadamou y Tzivinikos (2013).

La tabla 1 recoge el propósito y los resultados obtenidos en cada una de las investigaciones anteriores.

La literatura anterior muestra el gran interés por conocer qué información explica el riesgo de las acciones, y el último trabajo citado recoge el mismo interés pero teniendo en cuenta la 
Tabla 1

Revisión de literatura

\begin{tabular}{ll}
\hline Autor & Propósito \\
\hline Breen y Lerner (1973) & La investigación consiste en estudiar si \\
& las tradicionales variables explicativas \\
del riesgo explican los cambios de la \\
beta de la empresa durante el periodo \\
$1965-1970$
\end{tabular}

Lev y Kunitzky (1974)

Lev (1974)

Bildersee (1975)

Elgers y Murray (1982)

Ismail y Kim (1989)

Karpik y Belkaoui (1990)

Elyasiani y Mansur (2005)

Brimble y Hodgson (2007)
Investiga la relación entre riesgo y medidas de alisamiento

Investiga la relación entre el endeudamiento operativo y el riesgo de la acción

Analiza la relación entre la beta y las variables contables para determinar el impacto de la política de dividendos y la capacidad de la empresa para cubrir sus intereses y el dividendo correspondiente de las preferentes, en cada uno de los años de la muestra Investiga el impacto del índice de mercado en la relación entre el riesgo sistemático y la información contable

Estudia si la variable cash flow es capaz de mejorar la información que proporcionan los beneficios al explicar el riesgo de mercado

Investiga si el valor añadido explica mejor que otras variables el riesgo de la acción

Estudia la relación entre medidas del riesgo basadas en el mercado y ratios financieros obtenidos de la contabilidad, utilizando el modelo GARCH para estimar las betas Examina la asociación entre la información contable y varias medidas del riesgo sistemático, incluida la beta determinada mediante el modelo GARCH
Resultados

Las variables significativas no son siempre las mismas. La estabilidad del crecimiento de los beneficios, el tamaño, y el ratio payout presentan un coeficiente de la regresión principalmente negativo. La tasa de crecimiento y el número de acciones en el mercado presentan mayoritariamente un signo positivo. La relación de endeudamiento muestra una menor estabilidad en el signo de la relación que las otras variables

Las ventas, el dividendo y los gastos de capital, alisados, y el promedio del ratio payout son variables explicativas del riesgo sistemático de las acciones El endeudamiento operativo es una variable significativa explicativa del riesgo sistemático con signo negativo en 2 industrias: centrales eléctricas y la industria del acero

Los resultados sugieren que la relación es más consistente cuando se incorpora en el modelo información contable y no contable, que cuando únicamente se utilizan variables independientes de origen contable

El crecimiento de los activos, el endeudamiento financiero y el tamaño del activo explican el riesgo de mercado en los 3 índices de mercado utilizados

El cash flow contiene más información si se quiere explicar el riesgo

Los resultados muestran que el valor añadido proporciona más información de la beta de las acciones que la que ofrece el beneficio y el cash flow La relación entre las variables contables y la beta es más débil en las instituciones bancarias japonesas que en las de Estados Unidos

La mejor relación entre la información contable y la beta se obtiene cuando la beta se ha estimado utilizando el modelo M- GARCH 
Tabla 1 (continuación)

\begin{tabular}{|c|c|c|}
\hline Autor & Propósito & Resultados \\
\hline Agusman et al. (2008) & $\begin{array}{l}\text { Investiga la relación entre la } \\
\text { contabilidad y el riesgo del mercado de } \\
\text { capitales para una muestra de bancos } \\
\text { asiáticos utilizando los datos de panel }\end{array}$ & $\begin{array}{l}\text { Solamente el ratio reservas para } \\
\text { pérdidas crediticias/préstamos totales } \\
\text { es una variable significativa que } \\
\text { explica el riesgo sistemático de las } \\
\text { acciones de las empresas financieras }\end{array}$ \\
\hline $\begin{array}{l}\text { Campbell, Polk y } \\
\text { Vuolteenaho (2010) }\end{array}$ & $\begin{array}{l}\text { Analiza qué características de la } \\
\text { empresa predicen la tasa de } \\
\text { actualización de las acciones }\end{array}$ & $\begin{array}{l}\text { Los resultados muestran que los datos } \\
\text { contables deben jugar un papel } \\
\text { importante en determinar el coste de } \\
\text { capital de la empresa }\end{array}$ \\
\hline Menéndez et al. (2012) & $\begin{array}{l}\text { Estudia qué información explica el } \\
\text { riesgo sistemático de las acciones }\end{array}$ & $\begin{array}{l}\text { Los resultados muestran que la } \\
\text { información que explica el riesgo es } \\
\text { una combinación de variables } \\
\text { contables, información } \\
\text { macroeconómica e indicadores de } \\
\text { productividad }\end{array}$ \\
\hline $\begin{array}{l}\text { Papadamou y Tzivinikos } \\
\text { (2013) }\end{array}$ & $\begin{array}{l}\text { Estudia qué información explica el } \\
\text { riesgo sistemático y no sistemático de } \\
\text { las acciones de los bancos griegos } \\
\text { antes y después de la aplicación de las } \\
\text { NIIF (IFRS) }\end{array}$ & $\begin{array}{l}\text { Los resultados revelan que el tamaño es } \\
\text { una variable explicativa del riesgo de } \\
\text { las acciones después de la aplicación } \\
\text { de las NIIF, con signo negativo, y que } \\
\text { el ratio de endeudamiento, deuda a } \\
\text { largo plazo/fondos propios, no es una } \\
\text { variable estadísticamente significativa } \\
\text { ni antes ni después de la adopción de } \\
\text { las NIIF }\end{array}$ \\
\hline
\end{tabular}

Fuente: elaboración propia a partir de los trabajos de investigación indicados.

adopción de las NIIF. Como ya hemos dicho, conocer esta información facilita la gestión, pues permite anticipar los cambios en el riesgo y, por lo tanto, los cambios en el coste de capital o tasa de actualización.

No obstante, existe también un gran interés en la comunidad financiera por conocer los efectos de la aplicación de las NIIF bien en el coste de capital (Li, 2010) y, consecuentemente, en el riesgo, bien en la información contable (Landsman, Maydew y Thornock, 2012) o bien sobre la liquidez de las acciones (Christensen, Hail y Leuz (2013).

Dado que el coste de capital depende del riesgo, a continuación nos centraremos en los principales estudios que analizan los efectos de la aplicación de las NIIF en el coste de capital de las acciones.

Li (2010) muestra en su trabajo que en promedio la aplicación de las NIIF reduce el coste de capital de las acciones, y que el efecto es mayor en países con un sistema judicial fuerte y de calidad.

Landsman et al. (2012) muestran en su investigación que, en 16 países que adoptan las IFRS (NIIF), el contenido informativo del anuncio de beneficios se incrementa en relación con 11 países que mantienen los estándares contables domésticos, aunque el efecto de la aplicación de las NIIF depende de la fuerza legal en la aplicación de las nuevas normas de contabilidad.

Christensen et al. (2013) muestran con su investigación que existe una pequeña evidencia de que una voluntaria adopción de las NIIF significa un incremento en la liquidez de las acciones y una reducción en el coste de capital de los fondos propios, aunque los resultados son muy heterogéneos. 
Tabla 2

Técnicas de estudio empleadas

\begin{tabular}{lc}
\hline Estudio & Técnica de análisis \\
\hline Chen (2013) & Datos de panel estático \\
Christensen et al. (2013) & Datos de panel estático \\
Han y He (2013) & Datos de panel estático \\
Landsman et al. (2012) & Datos de panel estático \\
Menéndez et al. (2012) & Datos de panel estático \\
Papadamou y Tzivinikos (2013) & Datos de panel estático \\
Kim et al. (2014) & Modelo de regresión \\
Daske, Hail, Leuz y Verdi (2013) & Modelo de regresión \\
Li (2010) & Modelo de regresión \\
\hline
\end{tabular}

Fuente: elaboración propia a partir de los trabajos de investigación indicados.

Han y He (2013) muestran con su estudio que los cambios en el coste de capital de las empresas extranjeras en Estados Unidos se ven afectados por el uso de las NIIF en el país de origen.

Kim, Shi y Zhou (2014) muestran mediante el análisis de una muestra de 21,608 empresas de diferentes países que la adopción voluntaria de las NIIF reduce el coste de capital de las acciones sobre todo en países con instituciones financieras y legales sólidas.

La literatura existente tanto en el estudio de las variables que explican el riesgo de las acciones como en el estudio de los efectos de las NIFF en el coste de capital o en el mercado de capitales muestra una homogeneidad y una tendencia en la técnica de análisis empleada. En la tabla 2 recogemos los trabajos más recientes en ambos campos y la técnica de estudio utilizada.

La información de la tabla 2 muestra que recientemente las técnicas más utilizadas son el modelo de regresión y el estudio con datos de panel estático, lo que justifica, en parte, que nos hayamos decantado por la segunda técnica, ya que permite incorporar al modelo de regresión la variable tiempo, proporcionando los resultados una información más exacta y precisa de la muestra analizada.

La técnica de datos de panel presenta ventajas respecto a la regresión por mínimos cuadrados, tal y como indican Hsiao (2003) y Baltagi (2012). De acuerdo con los autores, la técnica de datos de panel proporciona al investigador más datos, incrementando los grados de libertad y reduciendo la colinealidad entre las variables explicativas. La técnica de datos de panel mejora la eficiencia de las estimaciones econométricas ${ }^{4}$.

\section{Diseño de la investigación}

El punto de partida del estudio es la estimación semestral del riesgo de las acciones (beta de mercado) de cada una de las 98 empresas que configuran la muestra. Medimos el riesgo utilizando el modelo CAPM, y calculamos, por lo tanto, la beta de mercado de las acciones calculando la covarianza entre la rentabilidad diaria de dichas acciones y la rentabilidad diaria del índice del mercado de referencia ${ }^{5}$. A continuación, mediante la técnica de datos de panel estático estudiamos

\footnotetext{
${ }^{4}$ Econometric Analysis of Panel Data, Badi H. Baltagi (cuarta edición), 2012.

5 Aunque la beta que estimamos se obtiene a partir del IBEX-35 con modelos de regresión por mínimos cuadrados ordinarios, conviene indicar que hemos realizado estimaciones alternativas utilizando otros índices (el Índice Total y el Índice General de la Bolsa de Madrid) y aproximaciones metodológicas alternativas (como los modelos auto regresivos EGARCH) sin obtener mejores resultados.
} 
la relación entre la beta de cada empresa y un amplio conjunto de variables independientes (que contienen información contable, financiera y del entorno macroeconómico).

En primer lugar estudiamos qué variables explicativas influyen en el riesgo sistemático de las acciones, utilizando para ello la muestra total y todo el periodo de estudio (2001-2011), pero como el objetivo principal es analizar el efecto de las NIIF sobre la relación entre la variable dependiente e independientes, a continuación incorporamos la variable dicotómica NIIF y posteriormente investigamos la relación entre las variables separando la muestra en 2 periodos temporales, 20012004, antes de la aplicación de las NIIF, y 2005-2011, después de la aplicación de las NIIF.

Para el desarrollo de la investigación utilizamos un total de 20 variables independientes, clasificables en 3 grandes bloques: a) VC o información obtenida directamente de la contabilidad (básicamente, del balance y de la cuenta de resultados); b) VCF o indicadores de tesorería, y c) VM o variables del entorno macroeconómico. En la tabla 3 presentamos cada variable e indicamos para cada una de ellas una referencia bibliográfica que justifica su utilización en el estudio.Con todo ello, postulamos el siguiente modelo:

$$
\beta_{i t}=\alpha_{0}+\sum_{j=1}^{J} \alpha c_{j} \cdot V C_{j i t}+\sum_{f=1}^{F} \alpha c f_{f} \cdot V C F_{f i t}+\sum_{m=1}^{M} \alpha m_{m} \cdot V M_{m t}+\mu_{i}+\varepsilon_{i t}
$$

donde: $\beta_{i t}$ es la medida del riesgo de mercado de las acciones de la empresa $i$ en el periodo $t$. $V C_{j i t}$ identifica al indicador contable $j$ obtenido de las cuentas anuales de la empresa $i$ en el periodo $t$. $V C F_{f i t}$ identifica la variable de tesorería $f$ de la empresa $i$ en el periodo $t$. $V M_{m t}$ identifica la variable de entorno macroeconómico $m$ para el periodo $t$.

La muestra comprende empresas representativas de todos los sectores económicos de la economía española, de las cuales 14 son financieras y 84 industriales. Trabajamos con información contable semestral y consideramos todos los semestres comprendidos en el periodo 2001-2011. Para deflactar las variables expresadas en valores absolutos se utilizaron 4 denominadores: valor contable de los fondos propios (VCFP), ventas (VENT), gastos financieros (GF) y activo total (AT).

En las siguientes tablas podemos observar la estadística descriptiva de cada una de las variables de la muestra. La tabla 4 incorpora la estadística descriptiva de las variables procedentes de la información contable.

En la tabla 5 encontramos la estadística descriptiva de las variables de tesorería.

En la tabla 6 se muestra la estadística descriptiva de las variables macroeconómicas.

La estadística descriptiva nos revela que la muestra investigada es una muestra en la que las empresas tienen una media de la rentabilidad de la explotación (ROA) del $4.58 \%$ y una rentabilidad media del accionista (ROE) del 8.46\%, siendo la desviación típica de esta última rentabilidad superior a la rentabilidad de la explotación. El margen de explotación, o beneficio antes de intereses e impuestos (BAIT)/VEN, variable estadísticamente significativa en todos los análisis realizados de la muestra de empresas industriales, tiene un valor medio igual al $8 \%$, siendo sin embargo la desviación típica muy elevada.

Vemos también que:

a) El ratio BAIT/GF tiene un valor promedio igual a 35,092, es decir, el BAIT es, en promedio, 35 veces superior a los gastos financieros.

b) El valor promedio del endeudamiento empresarial es igual a 1.336.

c) La solvencia de las empresas está representada por un valor promedio igual a 1.89.

d) El apalancamiento operativo de las empresas adquiere un valor promedio igual a 0.082 . 
Tabla 3

Descripción de las variables utilizadas

\begin{tabular}{|c|c|}
\hline Definición de la variable & Descripción \\
\hline $\mathrm{VC}$ & Información obtenida de las cuentas anuales \\
\hline Ratio de solvencia (SOL) (Brimble y Hodgson, 2007) & Activo corriente/Pasivo corriente \\
\hline $\begin{array}{l}\text { Ratio de endeudamiento (RE) (Brimble y Hodgson, } \\
\text { 2007) }\end{array}$ & $\begin{array}{l}\text { Deuda a largo plazo/(Fondos Propios + Pasivo a largo } \\
\text { plazo) }\end{array}$ \\
\hline Tamaño (TA) (Lee y Jang, 2007) & Logaritmo neperiano del activo total \\
\hline Payout (PA) (Elmoatasem, 2005) & Dividendo/Beneficio neto \\
\hline Crecimiento (CR) (Brimble y Hodgson, 2007) & $\begin{array}{l}\text { Logaritmo neperiano de la ratio: (Total de activos al final } \\
\text { de } \mathrm{t} \text { )/(Total de activos al final de } \mathrm{t}-1 \text { ) }\end{array}$ \\
\hline Valor añadido bruto (VAB) (Karpik y Belkaoui, 1990) & (Ingresos - Consumos intermedios) \\
\hline Valor añadido neto (VAN) (Karpik y Belkaoui, 1990) & (Valor añadido bruto - Dotaciones a la amortización) \\
\hline $\begin{array}{l}\text { Beneficio de la explotación (BAIT) (Campbell et al., } \\
\text { 2010) }\end{array}$ & Beneficio antes de intereses e impuestos \\
\hline Beneficio neto (BN) & Beneficio neto \\
\hline $\begin{array}{l}\text { Apalancamiento operativo (EO) (Lord, 1996; Brimble y } \\
\text { Hodgson, 2007) }\end{array}$ & BAIT/Ventas \\
\hline Apalancamiento financiero (EF) & Beneficio neto/BAIT \\
\hline VCF & Variables de tesorería \\
\hline Tesorería 1 (CF1) (Da y Warachka, 2009; Sloan, 1996) & $\begin{array}{l}\text { Flujo libre de tesorería: Resultado de la } \\
\text { explotación + Dotación a la amortización + Ingresos } \\
\text { financieros - Gastos financieros (+/-) variación de } \\
\text { deudores (+/-) variación de existencias (-/+) variación de } \\
\text { proveedores }\end{array}$ \\
\hline Tesorería 2 (CF2) & $\begin{array}{l}\text { CF1 - Variación inversiones en inmovilizado por } \\
\text { reposición }\end{array}$ \\
\hline Tesorería 3 (CF3) & CF1 - Dotación a la amortización \\
\hline VM & Variables de entorno macroeconómico \\
\hline Euribor (EUR) (Arfaoui y Abaoub, 2010) & $\begin{array}{l}\text { Precio del dinero a corto plazo fijado en el mercado } \\
\text { interbancario europeo }\end{array}$ \\
\hline Tipo de interés legal (TIL) (Arfaoui y Abaoub, 2010) & Tipo de interés básico de la deuda pública \\
\hline $\begin{array}{l}\text { Índice de precios de consumo (IPC) (Gosnell y } \\
\text { Nejadmalayeri, 2010) }\end{array}$ & Tasa de variación del índice de precios al consumo \\
\hline $\begin{array}{l}\text { Tasa de desempleo (TD) (Gosnell y Nejadmalayeri, } \\
\text { 2010) }\end{array}$ & Tasa de desempleo \\
\hline $\begin{array}{l}\text { Variación del producto interior bruto (PIB) (Vassalou, } \\
\text { 2003) }\end{array}$ & Tasa de variación del PIB \\
\hline Índice Dow Jones (DJ) (Hamao, Masulis y Ng, 1990) & Índice bursátil del mercado norteamericano \\
\hline
\end{tabular}

Fuente: elaboración propia a partir de los trabajos de investigación indicados.

e) El promedio del tamaño empresarial de la muestra es de 14, siendo la desviación típica de 2.45.

f) La tesorería empresarial, cash flow, tiene un valor promedio siempre positivo independientemente del denominador utilizado. Así, por ejemplo, el valor promedio del ratio CF1/AT es igual a $29.49 \mathrm{y}$ el valor medio que identifica a toda la muestra del ratio CF1/VCFP es igual a 78.75 .

g) El valor promedio que identifica a la variable tasa de desempleo es igual a 13.10.

h) El valor promedio de la variación del producto interior bruto (PIB) es de 1.11.

i) La media del Euribor toma un valor igual a 2.73, siendo la desviación típica muy reducida. 
Tabla 4

Estadística descriptiva variables obtenidas de las cuentas anuales $(\mathrm{n}=1,906)$

\begin{tabular}{lllll}
\hline Variable & Media & DE & Min & Máx \\
\hline SOL & 1.8940 & 7.8440 & 0.0226 & 267.0189 \\
RE & 1.3359 & 7.9540 & -179.8258 & 275.4222 \\
TA & 14.008 & 2.4488 & 3.5911 & 24.76842 \\
CR & 0.01374 & 1.7618 & -13.3585 & 10.0068 \\
EO & 0.0824 & 3.3323 & -68.55 & 69.5 \\
EF & -0.7329 & 40.1526 & -1447.045 & 161.28 \\
VAB/VCFP & 0.0269 & 1.2953 & -15.1743 & 43.2561 \\
VAN/VCFP & -0.0369 & 1.5555 & -16.1654 & 53.2561 \\
BAIT/VCFP & 0.1628 & 0.2859 & -0.8294 & 5.1508 \\
BN/VCFP & 0.0845 & 1.2560 & -13.9227 & 32.3561 \\
VAB/VENT & -0.0367 & 2.8038 & -74.55 & 26.2127 \\
VAN/VENT & -0.1569 & 3.8038 & -79.7095 & 16.2766 \\
BAIT/VENT & 0.0807 & 2.9314 & -68.55 & 69.5 \\
BN/VENT & 0.4302 & 4.6396 & -56.2857 & 74.4261 \\
VAB/AT & -0.0169 & 0.1449 & -1.3533 & 1.1515 \\
VAN/AT & -0.0326 & 0.1451 & -1.3533 & 1.0985 \\
BAIT/AT & 0.0458 & 0.1011 & -0.8940 & 1.3155 \\
BN/AT & 0.0311 & 0.0823 & -1.2784 & 0.8236 \\
VAB/GF & 21.1703 & 565.0327 & -450.2137 & 18363.5 \\
VAN/GF & 11.6983 & 536.9527 & -516.9695 & 18363.5 \\
BAIT/GF & 35.0928 & 590.6011 & -114.6694 & 18363.5 \\
BN/GF & 34.5037 & 592.2132 & -117.6861 & 18526 \\
\hline AT/ & & & & \\
\hline
\end{tabular}

AT: activo total; BAIT: beneficio antes de intereses e impuestos; BN: beneficio neto o beneficio del accionista; CR: crecimiento; EF: apalancamiento financiero; EO: apalancamiento operativo; GF: gastos financieros; RE: endeudamiento; SOL: solvencia; TA: tamaño, VAB: valor actual bruto; VAN: valor actual neto; VCFP: valor contable de los fondos propios; VENT: ventas.

Tabla 5

Estadística descriptiva variables obtenidas de las variables de tesorería $(\mathrm{N}=1906)$

\begin{tabular}{|c|c|c|c|c|}
\hline Variable & Media & Desv. típica & Min & Máx \\
\hline CF1/VCFP & 78.75621 & $2,917.615$ & -124.7762 & $110,673.7$ \\
\hline CF2/VCFP & 223.6988 & $7,906.332$ & -124.6212 & $299,736.3$ \\
\hline CF3/VCFP & 210.2871 & $7,901.489$ & -124.7892 & $299,736.3$ \\
\hline CF1/VENT & 101.0607 & $2,576.939$ & -568.6667 & $69,826.57$ \\
\hline CF2/VENT & 760.9844 & $23,336.23$ & -568.3333 & $862,209.8$ \\
\hline CF3/VENT & 186.7152 & $5,312.42$ & -571.6667 & $189,110.4$ \\
\hline CF1/AT & 29.48855 & $1,684.88$ & $-61,630.55$ & $18,102.24$ \\
\hline CF2/AT & 138.0153 & $4,005.131$ & -7.969925 & $144,635.3$ \\
\hline CF3/AT & 8.135573 & $2,065.987$ & $-61,630.55$ & $49,026.06$ \\
\hline CF1/GF & $4,574.034$ & $156,360.6$ & $-16,508$ & $546,119.2$ \\
\hline $\mathrm{CF} 2 / \mathrm{GF}$ & $12,592.06$ & $423,503.3$ & $-16,508$ & $1.48 \mathrm{e}+07$ \\
\hline CF3/GF & $12,281.62$ & $423,454.2$ & $-18,164$ & $1.48 \mathrm{e}+07$ \\
\hline
\end{tabular}

AT: activo total; CF1: flujo de tesorería de las operaciones; $\mathrm{CF} 2$ (CF1 \pm variaciones del flujo de tesorería por operaciones de inversión; CF3: CF1 - dotaciones a la amortización económica; GF: gastos financieros; VCFP: valor contable de los fondos propios; VENT: ventas. 
Tabla 6

Estadística descriptiva de las variables macroeconómicas $(\mathrm{n}=2,056)$

\begin{tabular}{lllll}
\hline Variable & Media & Desv. Típica & Min & Máx. \\
\hline EUR & 2.7341 & 0.6057 & 2.014 & 3.869 \\
TIL & 4.3336 & 0.5690 & 3.75 & 5.5 \\
IPC & 1.0834 & 1.0656 & -1.3 & 2.9 \\
TD & 13.1065 & 4.7385 & 7.95 & 22.85 \\
PIB & 1.1086 & 2.1423 & -4.5 & 3.734219 \\
DJ & -0.0171 & 0.0986 & -0.2384 & 0.1100 \\
S\&P500 & -0.01418 & 0.1109 & -0.2970 & 0.1388 \\
\hline
\end{tabular}

DJ: índice bursátil Dow Jones; EUR: Euribor; IPC: variación del índice de precios al consumo; PIB: variación del producto interior bruto; S\&P500: índice bursátil Standard \& Poor's; TD: tasa de desempleo; TIL: tipo de interés legal del dinero.

\section{Resultados}

En este apartado presentamos los mejores modelos obtenidos después de:

a) Seleccionar las variables independientes una a una mediante una regresión por mínimos cuadrados entre el riesgo sistemático y cada una de las variables independientes utilizadas en el estudio. Solo las variables estadísticamente significativas y no correlacionadas ${ }^{6}$ se han incorporado en el modelo.

b) Corregir la heterocedasticidad y la autocorrelación mediante las estimaciones realizadas con Panel Corrected Standard Errors (Wooldridge, 2002) ${ }^{7}$.

En el siguiente apartado hacemos referencia a los resultados de estudiar la conexión entre el riesgo de mercado de la acción, la beta, y las variables independientes utilizando todo el periodo de estudio, 2001-2011, y en el apartado «Análisis del efecto que ejerce la introducción de las NIIF» se muestran los resultados de analizar cómo influye la introducción de las NIIF en dicha relación.

\section{Análisis de la relación riesgo de la acción-información en el periodo 2001-2011}

Para este análisis utilizamos 3 muestras: la muestra total (que contiene 98 empresas), la de empresas financieras (con 14 empresas) y la de empresas industriales (con un total de 84 empresas). La tabla 7 recoge las mejores estimaciones para cada muestra de empresas.

El estudio desarrollado nos permite concluir que el riesgo de la acción se puede explicar mediante una combinación de variables contables y macroeconómicas aunque las variables no son siempre las mismas, pues varían según el tipo de empresa.

Así, para las empresas industriales las variables estadísticamente significativas que explican el riesgo son el tamaño, la relación BAIT/VEN, el Euribor, la tasa de desempleo, el tipo de interés legal y el PIB. Los resultados del estudio nos indican que la variable contable que tiene mayor poder explicativo del riesgo de la acción es el margen de explotación, BAIT/VEN. A mayor valor

\footnotetext{
${ }^{6}$ En el Apéndice 1 se presentan los coeficientes de correlación. También hemos utilizado la técnica de datos de panel para estudiar la relación entre el riesgo y cada una de las variables independientes que configuran la investigación, pero el estudio mediante la técnica de regresión nos ha proporcionado unos resultados con una $\mathrm{R}^{2}$ mejor.

7 Véase Beck y Katz (2001).
} 
Tabla 7

Estimación de los modelos con la muestra total

\begin{tabular}{|c|c|c|c|c|c|}
\hline Variable & Signo esperado & $\begin{array}{l}\text { Muestra total } \\
(\mathrm{n}=1,794)\end{array}$ & $\begin{array}{l}\text { Muestra de empresas } \\
\text { financieras }(n=222)\end{array}$ & $\begin{array}{l}\text { Muestra de empresas no } \\
\text { financieras }(n=1,572)\end{array}$ & \\
\hline$\alpha_{0}$ & & 9.67 & -0.93 & 6.43 & \\
\hline TA & + & $\begin{array}{l}3.57^{* * *} \\
(0.000)\end{array}$ & $\begin{array}{l}3.08^{* * *} \\
(0.002)\end{array}$ & $\begin{array}{l}1.61^{*} \\
(0.108)\end{array}$ & \\
\hline BAIT/VN & + & $\begin{array}{l}-4.68^{* * *} \\
(0.000)\end{array}$ & & $\begin{array}{l}-4.17^{* * *} \\
(0.000)\end{array}$ & \\
\hline BAIT/AT & - & & $\begin{array}{l}-2.13 \\
(0.034)\end{array}$ & & \\
\hline EUR & & $\begin{array}{l}-8.09^{* * * *} \\
(0.000)\end{array}$ & $\begin{array}{l}-2.33^{*} \\
(0.0021)\end{array}$ & $\begin{array}{l}-7.38^{* * * *} \\
(0.000)\end{array}$ & \\
\hline $\mathrm{TD}$ & & + & $\begin{array}{l}-27.69^{* * *} \\
(0.004)\end{array}$ & $\begin{array}{l}-5.58^{* * * *} \\
(0.000)\end{array}$ & $\begin{array}{l}-5.58^{* * *} \\
(0.000)\end{array}$ \\
\hline TIL & + & & & $\begin{array}{l}2.22^{*} \\
(0.026)\end{array}$ & \\
\hline PIB & - & & $\begin{array}{l}-1.92^{*} \\
(0.057)\end{array}$ & $\begin{array}{l}-4.90^{* * * *} \\
(0.000)\end{array}$ & \\
\hline IPC & + & & $\begin{array}{l}2.13^{*} \\
(0.035)\end{array}$ & & \\
\hline DJ & \pm & $\begin{array}{l}-2.89^{* * *} \\
(0.004)\end{array}$ & & & \\
\hline $\mathrm{R}^{2}$ & 0.2528 & 0.424 & 0.2731 & & \\
\hline Prob $>F$ & 0.0000 & 0.0000 & 0.0000 & & \\
\hline Test Hausman & 96.78 & 61.51 & 46.60 & & \\
\hline \multirow[t]{2}{*}{ Prob $>\mathrm{chi}^{2}$} & 0.0000 & 0.0000 & 0.0000 & & \\
\hline & Efectos fijos & Efectos fijos & Efectos fijos & & \\
\hline
\end{tabular}

BAIT/AT: beneficio de la explotación relacionado con el activo total, rentabilidad de la explotación; BAIT/VEN: beneficio de la explotación relacionado con las ventas; DJ: Dow Jones índice bursátil; EUR: Euribor; IPC: variación del índice de precios de consumo; PIB: producto interior bruto; TA: tamaño; TD: tasa de desempleo; TIL: tipo de interés legal.

Fuente: elaboración propia.

* La variable es significativa al $10 \%$.

** La variable es significativa al $5 \%$.

*** La variable es significativa al $1 \%$.

del indicador, menos riesgo sistemático presentan las acciones. La otra variable contable que explica el riesgo es el tamaño, y los resultados muestran una relación positiva entre el riesgo y el tamaño de la empresa, resultado que concuerda con el obtenido en Brimble y Hodgson (2007) y en Menéndez et al. (2012).

Las otras variables que explican el riesgo de las acciones industriales son macroeconómicas. Los resultados muestran que el riesgo sistemático de la acción se reduce con un aumento del Euribor, del PIB y de la tasa de desempleo. Las 3 variables son altamente significativas desde el punto de vista estadístico, pero el resultado que más sorprende es la relación entre la beta de la acción y la tasa de desempleo, una relación de signo negativo. No obstante, si tenemos en cuenta que la beta o el riesgo sistemático es un cálculo de la variabilidad de la rentabilidad de la acción respecto al índice bursátil, es posible encontrar que a mayor tasa de desempleo, lo cual coincide con un escenario de no crecimiento económico, la volatilidad de las acciones sea menor. Anticipándonos a los resultados posteriores, se observa que esta variable es siempre altamente significativa y con signo negativo, independientemente del periodo analizado. 
La $\mathrm{R}^{2}$ adquiere un valor elevado, 0.2731 , lo cual justifica la bondad del modelo y de los resultados.

Los resultados obtenidos del estudio de la muestra de empresas financieras son algo diferentes respecto a las empresas industriales; así, las únicas variables contables que explican el riesgo de las acciones financieras es el tamaño y el ratio BAIT/AT. Ambas son variables estadísticamente significativas, aunque el tamaño tiene más poder explicativo, y los resultados revelan una relación positiva entre el riesgo y el tamaño empresarial y entre el riesgo y el ratio BAIT/AT, debido quizá a que un mayor BAIT en la empresa financiera es resultado de un mayor endeudamiento empresarial. Hay que tener en cuenta que el estudio se realiza sobre un amplio periodo de tiempo caracterizado por un elevado crecimiento y el posterior estallido de la burbuja inmobiliaria.

Las otras variables que explican el riesgo de las acciones son el Euribor, la tasa de desempleo, el PIB y el índice de precios al consumo. Aunque las 4 variables explican el riesgo, la variable independiente con mayor poder explicativo es la tasa de desempleo. El riesgo sistemático de las acciones, o beta, se reduce con un incremento del Euribor y de la tasa de desempleo y se incrementa con un aumento del PIB y del índice de precios al consumo.

El estudio de las empresas financieras proporciona el modelo con la $\mathrm{R}^{2}$ más elevada y, por lo tanto, desde el punto de vista estadístico, el mejor modelo o conexión entre variable dependiente y las variables explicativas del riesgo.

El estudio de la muestra total, las 98 empresas, revela unos resultados similares a los obtenidos en el estudio de las empresas industriales. Así, el tamaño, el ratio de eficiencia BAIT/VEN, el Euribor y la tasa de desempleo explican el riesgo de la acción, al igual que para la muestra de empresas industriales. No obstante, aparece el índice bursátil Dow Jones como variable explicativa del riesgo, de forma que si el Dow Jones se incrementa, se reduce el riesgo de las acciones españolas.

La investigación realizada pone de manifiesto que la mayor parte de la información contable utilizada como variable independiente no tiene poder explicativo del riesgo sistemático de la acción; sorprendentemente, ni el endeudamiento, ni la rentabilidad de los accionistas juegan un papel importante desde el punto de vista estadístico, aunque este resultado coincide con el obtenido en trabajos anteriores (Papadamou y Tzivinikos, 2013; Menéndez et al., 2012, Brimble y Hodgson, 2007).

\section{Análisis del efecto que ejerce la introducción de las NIIF}

En este apartado estudiamos si la introducción de las NIIF afecta a la conexión entre el riesgo de las acciones y las variables explicativas. Para ello primero introducimos una variable dicotómica, NIIF, que toma valor 1 para los ejercicios 2005-2011 y 0 para los años 2001 a 2004. Posteriormente analizamos los efectos de las NIIF, pero estudiando por separado los 2 periodos: 2001-2004 y 2005-2011.

La incorporación de la variable dicotómica está justificada mediante la realización del test de Chow $^{8}$, que nos revela los siguientes resultados ${ }^{9}$ :

$$
\text { F-statistic }=122.98, \text { prob }>F=0.0000
$$

\footnotetext{
8 Greene (2012) y Pulido y Pérez (2001).

9 STATA. 12
} 
Tabla 8

Estimación de los modelos con la variable dicotómica

\begin{tabular}{|c|c|c|c|c|}
\hline Variable & Signo & $\begin{array}{l}\text { Muestra total } \\
(\mathrm{n}=1,779)\end{array}$ & $\begin{array}{l}\text { Muestra de empresas } \\
\text { financieras }(n=222)\end{array}$ & $\begin{array}{l}\text { Muestra de empresas no } \\
\text { financieras }(n=1,572)\end{array}$ \\
\hline$\alpha_{0}$ & & 10.77 & -6.47 & 4.40 \\
\hline TA & + & $\begin{array}{l}2.90^{* * *} \\
(0.004)\end{array}$ & $\begin{array}{l}13.22^{* * *} \\
(0.000)\end{array}$ & $\begin{array}{l}2.69^{* * * *} \\
(0.007)\end{array}$ \\
\hline BAIT/VEN & - & $\begin{array}{l}-3.10^{\text {**** }} \\
(0.002)\end{array}$ & & $\begin{array}{l}-3.07^{\text {***** }} \\
(0.002)\end{array}$ \\
\hline BAIT/AT & - & & $\begin{array}{l}1.13 \\
(0.260)\end{array}$ & \\
\hline EUR & + & $\begin{array}{l}-10.32^{\text {**** }} \\
(0.000)\end{array}$ & $\begin{array}{l}-3.09^{* * *} \\
(0.002)\end{array}$ & $\begin{array}{l}-9.74^{* * * *} \\
(0.000)\end{array}$ \\
\hline $\mathrm{TD}$ & + & $\begin{array}{l}-27.68^{\text {***** }} \\
(0.000)\end{array}$ & $\begin{array}{l}-5.22^{* * *} \\
(0.000)\end{array}$ & $\begin{array}{l}-19.93^{* * * *} \\
(0.000)\end{array}$ \\
\hline TIL & + & & & $\begin{array}{l}1.21 \\
(0.226)\end{array}$ \\
\hline PIB & - & & $\begin{array}{l}1.40 \\
(0.161)\end{array}$ & $\begin{array}{l}4.76^{* * * *} \\
(0.000)\end{array}$ \\
\hline IPC & + & & $\begin{array}{l}1.65^{*} \\
(0.100)\end{array}$ & \\
\hline DJ & \pm & $\begin{array}{l}-1.16 \\
(0.246)\end{array}$ & & \\
\hline NIIF & - & $\begin{array}{l}9.09^{* * * *} \\
(0.000)\end{array}$ & $\begin{array}{l}-1.87^{*} \\
(0.062)\end{array}$ & $\begin{array}{l}9.67^{* * * *} \\
(0.000)\end{array}$ \\
\hline R2 & & 0.2782 & 0.5634 & 0.3159 \\
\hline Prob $>F$ & & 0.0000 & 0.0000 & 0.0000 \\
\hline Test Hausman & & -30.58 & 34.56 & 9.0 \\
\hline $\mathrm{Chi}^{2}$ & & $<0.0000$ & $<0.0000$ & 0.2475 \\
\hline Prob $>\mathrm{Chi}^{2}$ & & Efectos fijos & Efectos fijos & Efectos aleatorios \\
\hline
\end{tabular}

BAIT/AT: beneficio de la explotación relacionado con el activo total, rentabilidad de la explotación; EUR: Euribor; IPC: variación del índice de precios de consumo; TA: tamaño; TD: tasa de desempleo.

Fuente: elaboración propia.

* La variable es significativa al $10 \%$.

** La variable es significativa al $5 \%$.

*** La variable es significativa al $1 \%$.

Por lo tanto, se muestra la existencia de un cambio estructural a partir del 2005 que justifica la variable dummy y el estudio de la muestra en 2 periodos diferentes, antes y después de 2005 .

\section{Estudio del efecto de la aplicación de las NIIF mediante variables dicotómicas}

En este apartado analizamos si la relación entre el riesgo sistemático de la acción y la información es diferente para el periodo 2005-2011, en el que las empresas cotizadas aplican las NIIF. El resultado de esta estimación se presenta en la tabla 8.

Los resultados obtenidos nos dicen que la aplicación de las NIIF sí afecta a la relación entre el riesgo sistemático de las acciones y las variables explicativas. La variable dicotómica es significativa en el estudio de las 3 muestras, siendo más significativa para la muestra total y para la muestra de empresas industriales o no financieras. 
El estudio de la muestra de empresas industriales revela que la aplicación de las NIIF comporta:

a) Un incremento en el riesgo de las acciones, pues la variable dicotómica muestra un signo positivo.

b) Las variables explicativas del riesgo mantienen en términos generales su significación estadística. Solo el tamaño aumenta el poder explicativo, y el tipo de interés legal deja de ser una información explicativa del riesgo.

c) El signo de la relación se mantiene, excepto para el PIB, que ahora muestra una relación positiva con el riesgo.

La bondad estadística del modelo mejora, pues la $\mathrm{R}^{2}$ obtenida en este caso es superior a la obtenida sin la incorporación de la variable dual.

El análisis de la muestra de empresas financieras revela que:

a) El riesgo de las acciones se reduce, pues la variable binaria muestra un signo negativo.

b) Las variables explicativas del riesgo se mantienen, aunque hay algún cambio de nivel de significación estadística. Así, el Euribor incrementa el poder explicativo y el PIB deja de ser una variable explicativa.

c) El riesgo de las acciones se incrementa con el tamaño y el índice de precios al consumo y se reduce con el Euribor y la tasa de desempleo.

d) Se incrementa la bondad del modelo al obtener una $\mathrm{R}^{2}$ más elevada que en el estudio anterior, sin variable dummy.

e) De nuevo es la muestra de empresas financieras la que nos permite obtener el mejor modelo explicativo.

El estudio de todas las empresas a la vez no revela grandes cambios, pues las variables explicativas del riesgo son prácticamente las mismas que sin la variable binaria; solo el índice bursátil Dow Jones deja de ser una variable explicativa del riesgo.

\section{Estudio de la influencia de las NIIF dividiendo el periodo 2001-2011 en 2 períodos}

Con el fin de exprimir al máximo la muestra hemos analizado la relación entre la variable dependiente y las variables independientes dividiendo el periodo de estudio, 2001-2011, en 2 periodos: a) desde 2001 hasta 2004, y b) desde 2005 hasta 2011. En este apartado mostramos los resultados obtenidos de dicho estudio. En la tabla 9 presentamos los resultados del estudio del periodo 2001-2004, y en la tabla 10 mostramos los resultados del estudio del periodo 2005-2011.

Los datos de la tabla 9 muestran que la relación entre el riesgo de las acciones y la información contable y macroeconómica es solo ligeramente diferente en el periodo 2001-2004 respecto al periodo total, pues se observan algunos cambios en el nivel de significación estadística. Se observa que en el periodo 2001-2004, antes de la aplicación de las NIIF, las variables que aparecen como explicativas del riesgo son prácticamente las mismas que en el primer estudio, aunque se perciben ciertos cambios:

a) En el análisis de la totalidad de la muestra se reduce el poder explicativo de las variables tasa de desempleo y Dow Jones, aunque se mantienen los signos de la relación. La $\mathrm{R}^{2}$ obtenida 
Tabla 9

Análisis econométrico del periodo 2001-2004

\begin{tabular}{|c|c|c|c|c|}
\hline Variable & Signo esperado & $\begin{array}{l}\text { Muestra total } \\
(\mathrm{n}=642)\end{array}$ & $\begin{array}{l}\text { Muestra de empresas } \\
\text { financieras }(n=98)\end{array}$ & $\begin{array}{l}\text { Muestra de empresas no } \\
\text { financieras }(n=544)\end{array}$ \\
\hline$\alpha_{0}$ & & 2.69 & 0.78 & 2.45 \\
\hline TA & + & $\begin{array}{l}3.62^{* * *} \\
(0.000)\end{array}$ & $\begin{array}{l}-1.58^{*} \\
(0.119)\end{array}$ & $\begin{array}{l}2.76^{* * * *} \\
(0.006)\end{array}$ \\
\hline BAIT/VEN & - & $-3.57^{* * * *}(0.000)$ & & $\begin{array}{l}-2.97^{\text {***** }} \\
(0.003)\end{array}$ \\
\hline BAIT/AT & - & & $\begin{array}{l}-0.58 \\
(0.561)\end{array}$ & \\
\hline EUR & + & $\begin{array}{l}-2.99^{* * * *} \\
(0.003)\end{array}$ & $-2.71^{* * *}$ & $\begin{array}{l}-1.06 \\
(0.289)\end{array}$ \\
\hline $\mathrm{TD}$ & + & $\begin{array}{l}-2.48^{* * *} \\
(0.013)\end{array}$ & $\begin{array}{l}1.07 \\
(0.289)\end{array}$ & $\begin{array}{l}-3.16^{\text {***** }} \\
(0.002)\end{array}$ \\
\hline TIL & + & & & $\begin{array}{l}-1.86^{*} \\
(0.063)\end{array}$ \\
\hline PIB & & & $\begin{array}{l}2.37^{*} \\
(0.020)\end{array}$ & $\begin{array}{l}-0.25 \\
(0.802)\end{array}$ \\
\hline IPC & & & $\begin{array}{l}2.50^{*} \\
(0.014)\end{array}$ & \\
\hline DJ & & $\begin{array}{l}-1.62^{*} \\
(0.105)\end{array}$ & & \\
\hline $\mathrm{R} 2$ & & 0.1061 & 0.529 & 0.1216 \\
\hline Prob $>F$ & & 0.0000 & 0.0000 & 0.0000 \\
\hline Test Hausman & & 2.90 & 13.57 & 22.74 \\
\hline $\mathrm{Chi}^{2}$ & & 0.7161 & 0.0348 & 0.0009 \\
\hline \multicolumn{5}{|l|}{ Prob $>\mathrm{chi}^{2}$} \\
\hline & & Efectos aleatorios & Efectos fijos & Efectos fijos \\
\hline
\end{tabular}

BAIT/AT: beneficio de la explotación relacionado con el activo total, rentabilidad de la explotación; EUR: Euribor; IPC: variación del índice de precios de consumo; TA: tamaño; TD: tasa de desempleo.

Fuente: elaboración propia.

* La variable es significativa al $10 \%$.

** La variable es significativa al $5 \%$.

*** La variable es significativa al $1 \%$.

es inferior que la obtenida en el estudio del apartado «Análisis de la relación riesgo de la acción-información en el periodo 2001-2011».

b) En el estudio de las empresas industriales el tamaño gana poder explicativo y el Euribor y el PIB dejan de ser variables explicativas del riesgo, y la $\mathrm{R}^{2}$ obtenida también es inferior a la obtenida en el primer estudio.

c) En el estudio de las empresas financieras el tamaño, el ratio BAIT/AT y la tasa de desempleo pierden significación estadística, hasta el punto de que el ratio BAIT/AT y la tasa de desempleo dejan de ser significativas. Gana poder explicativo el Euribor y mantienen su significación estadística el índice de precios al consumo y el PIB. La $\mathrm{R}^{2}$ conseguida es superior a la obtenida en el primer estudio y ligeramente inferior al análisis con variable dicotómica.

Los datos de la tabla 10, estudio del periodo 2005-2011, muestran que los modelos obtenidos entre el riesgo sistemático de las acciones y las variables explicativas presentan las $\mathrm{R}^{2}$ más elevadas 
Tabla 10

Análisis econométrico periodo 2005-2011

\begin{tabular}{|c|c|c|c|c|}
\hline Variable & Signo esperado & $\begin{array}{l}\text { Muestra total } \\
(\mathrm{n}=1,133)\end{array}$ & $\begin{array}{l}\text { Muestra de empresas } \\
\text { financieras } \\
(\mathrm{n}=124)\end{array}$ & $\begin{array}{l}\text { Muestra de empresas no } \\
\text { financieras }(n=1,009)\end{array}$ \\
\hline$\alpha_{0}$ & & 8.63 & -3.93 & 6.16 \\
\hline $\mathrm{TA}$ & + & $\begin{array}{l}-1.00 \\
(0.316)\end{array}$ & $\begin{array}{l}7.56^{* * * *} \\
(0.000)\end{array}$ & $\begin{array}{l}-1.98^{*} \\
(0.049)\end{array}$ \\
\hline BAIT/VEN & - & $\begin{array}{l}-2.98^{* * *} \\
(0.003)\end{array}$ & & $\begin{array}{l}-3.02^{* * *} \\
(0.003)\end{array}$ \\
\hline BAIT/AT & - & & $\begin{array}{l}-2.05^{*} \\
(0.041)\end{array}$ & \\
\hline EUR & + & $\begin{array}{l}-9.40^{* * *} \\
(0.000)\end{array}$ & $\begin{array}{l}-2.85^{* *} \\
(0.004)\end{array}$ & $\begin{array}{l}-8.25^{* * *} \\
(0.000)\end{array}$ \\
\hline TD & + & $\begin{array}{l}-29.41^{* * *} \\
(0.000)\end{array}$ & $\begin{array}{l}-5.88^{* * * *} \\
(0.000)\end{array}$ & $\begin{array}{l}-21.17^{\text {**** }} \\
(0.000)\end{array}$ \\
\hline TIL & + & & & $\begin{array}{l}2.46^{* *} \\
(0.014)\end{array}$ \\
\hline PIB & & & $\begin{array}{l}2.16^{*} \\
(0.031)\end{array}$ & $\begin{array}{l}7.31^{* * *} \\
(0.000)\end{array}$ \\
\hline DJ & & $\begin{array}{l}-1.88^{*} \\
(0.060)\end{array}$ & & \\
\hline $\mathrm{R} 2$ & & 0.3330 & 0.5589 & 0.3683 \\
\hline Prob $>F$ & & 0.0000 & 0.0000 & 0.0000 \\
\hline Test Hausman & & 98.00 & 3.74 & 39.94 \\
\hline $\mathrm{Chi}^{2}$ & & 0.0000 & 0.5874 & 0.0000 \\
\hline Prob $>\mathrm{Chi}^{2}$ & & Efectos fijos & Efectos aleatorios & Efectos fijos \\
\hline
\end{tabular}

BAIT/AT: beneficio de la explotación relacionado con el activo total, rentabilidad de la explotación; EUR: Euribor; IPC: variación del índice de precios de consumo; TA: tamaño; TD: tasa de desempleo.

Fuente: elaboración propia.

* La variable es significativa al $10 \%$.

** La variable es significativa al $5 \%$.

*** La variable es significativa al $1 \%$.

de toda la investigación, excepto para la muestra de empresas financieras, que es ligeramente inferior a la obtenida en el estudio con variable dicotómica.

El análisis del periodo 2005-2011 revela que las variables que explican el riesgo sistemático de las acciones es siempre una combinación de variables contables y macroeconómicas, y que la información varía ligeramente entre empresas industriales y empresas financieras.

Los resultados manifiestan que:

a) Si utilizamos todas las empresas, sin considerar si son o no financieras, las variables que explican el riesgo son el BAIT/VEN, el Euribor, la tasa de desempleo y el índice bursátil Dow Jones. Las 3 primeras con un elevado poder explicativo, y todas ellas con un signo negativo en la relación. La diferencia más importante es que ahora el tamaño no es una variable explicativa del riesgo, tanto si lo comparamos con el estudio inicial como si lo hacemos con el estudio realizado con variable binaria. 
b) Si estudiamos la muestra de empresas industriales vemos que el tamaño pierde significación estadística y cambia el signo de la relación y el tipo de interés legal gana poder explicativo del riesgo con signo positivo, todo ello si comparamos los modelos con los resultados obtenidos al introducir la variable binaria NIIF.

El análisis de la muestra de empresas financieras revela que el tamaño mantiene su poder explicativo, así como la tasa de desempleo. Aparecen el BAIT/AT (rentabilidad de la explotación) y el PIB como variables explicativas, aunque ambas variables ya lo eran en el estudio inicial (apartado «Análisis de la relación riesgo de la acción-información en el periodo 2001-2011»).

\section{Conclusiones}

La investigación empírica desarrollada en este estudio demuestra que una combinación de información contable y macroeconómica explica el riesgo sistemático de las acciones, aunque las variables que explican el riesgo varían según el tipo de empresa (financiera o no financiera) y según el periodo analizado.

El riesgo sistemático de las acciones de las empresas industriales está relacionado siempre, en todos los análisis realizados, con el tamaño (TA), con el ratio BAIT/VEN (margen de la explotación), con el Euribor (EUR), con la tasa de desempleo (TD) con el tipo de interés legal (TIL) y con el producto interior bruto (PIB). En todos los análisis realizados el tamaño muestra en general un signo positivo en la relación, y el ratio BAIT/VEN, el Euribor y la tasa de desempleo muestran un signo negativo. El TIL muestra una relación positiva con la variable dependiente en el estudio total y en el estudio del periodo 2005-2011 y una relación negativa y significativa en el periodo 2001-2004. Y el PIB revela una relación negativa en el análisis inicial, todo el periodo, pero positiva al introducir la variable NIIF y al estudiar el periodo 2005-2011.

Así pues, podemos concluir que el riesgo sistemático de una acción industrial se puede estimar observando 6 informaciones: 2 variables contables —el tamaño y el ratio BAIT/VEN (margen de la explotación)_ y 4 variables macroeconómicas —Euribor, tasa de desempleo, TIL y PIB—.

Si tenemos en cuenta que la inmensa mayoría de las PYME son empresas industriales, estos resultados son de gran ayuda para poder medir objetivamente el riesgo de mercado de la actividad empresarial que no cotiza en la bolsa de valores.

Por lo tanto, para medir el riesgo de una acción de forma objetiva hay que tener en cuenta, además de la información macroeconómica, el tamaño de la empresa y el ratio BAIT/VEN. Así pues, 2 empresas del mismo sector económico y tamaño similar pueden tener riesgo diferente y, consecuentemente, un coste de capital diferente según el margen de la explotación obtenido, ratio BAIT/VEN.

El riesgo sistemático de las acciones de las empresas financieras viene determinado o explicado por el tamaño empresarial (TA), por el indicador BAIT/AT (ROA), por el Euribor (EUR), por la tasa de desempleo (TD), por el PIB y por el índice de precios al consumo (IPC): 2 variables contables y 4 macroeconómicas. El tamaño y el Euribor muestran una estabilidad a lo largo de los diferentes estudios en su significación estadística y en el signo de la relación. No ocurre lo mismo con los otros indicadores; así, el ROA es una variable significativa y positiva cuando estudiamos todo el periodo, pero con signo negativo en el análisis del periodo 2005-2011; la tasa de desempleo es siempre significativa con signo negativo, excepto en el periodo 2001-2004, en el cual no es una variable estadísticamente significativa. Respecto al PIB, esta información es siempre una variable con poder explicativo del riesgo con signo positivo, excepto en el estudio realizado con variable 
binaria, y el índice de precios al consumo es siempre una variable estadísticamente significativa con signo positivo, excepto en el periodo de estudio 2005-2011.

El estudio de las empresas financieras es el que nos ha proporcionado siempre los modelos con la $\mathrm{R}^{2}$ más elevada.

El estudio del efecto de las NIIF mediante la variable dicotómica refleja que las variables que explican el riesgo sistemático son las mismas tanto para la muestra de empresas financieras como para la muestra de empresas industriales que los obtenidos en el estudio inicial, todo el periodo. Se observa alguna pequeña modificación en el nivel de significación estadística, pero no se producen cambios relevantes. La variable dicotómica es significativamente estadística y positiva para las empresas industriales, lo cual indica que se manifiesta un incremento en el riesgo de las acciones de estas empresas en el periodo 2005-2011. No obstante, la variable binaria de las empresas financieras, que también es significativa, presenta un signo negativo, lo cual quiere decir que con la aplicación de las NIIF se observa una reducción en el riesgo sistemático de las acciones financieras.

El estudio del efecto de las NIIF separando la muestra en 2 períodos, 2001-2004 y 2005-2011, refleja que para la muestra de empresas financieras la relación entre la variable dependiente e independientes es prácticamente la misma: existen muy pocas diferencias. En el periodo 20012004 aparecen 4 variables estadísticamente significativas: el tamaño, el Euribor, el PIB y el IPC, mientras que en el periodo 2005-2011 las variables con poder explicativo son el tamaño, el ratio BAIT/AT, el Euribor, la tasa de desempleo y el PIB. En el primer periodo, 4 variables explican el riesgo; en el segundo periodo, 5 variables explican el riesgo, que salvo por el IPC son las mismas variables que obtuvimos como variables explicativas del riesgo sistemático al estudiar todo el periodo temporal (2001-2011).

El modelo obtenido para el periodo 2005-2011 muestra una $\mathrm{R}^{2}$ ligeramente superior a la $\mathrm{R}^{2}$ obtenida en el análisis del periodo 2001-2004.

Para las empresas industriales, el estudio de los 2 periodos temporales evidencia algunas diferencias. En el periodo 2005-2011, 6 variables explican el riesgo sistemático de la acción: el tamaño, el ratio BAIT/VEN, el Euribor, la tasa de desempleo, el TIL y el PIB, mientras que en el 2001-2004 solo 4 variables explican el riesgo. Los resultados obtenidos del estudio del periodo 2005-2011 muestran una $\mathrm{R}^{2}$ mucho mejor (0.3683) que la obtenida en el estudio del periodo 2001-2004 (0.1216).

En resumen, podemos concluir que:

a) El riesgo de las acciones industriales viene determinado por el tamaño, el ratio BAIT/VEN (margen de explotación), el Euribor, la tasa de desempleo, el y el PIB.

b) El riesgo de las acciones financieras viene determinado por el tamaño, el BAIT/AT (rentabilidad de la explotación), el Euribor, la tasa de desempleo, el PIB y el IPC.

c) El tamaño, el Euribor, la tasa de desempleo y el PIB son comunes a los 2 tipos de empresas.

d) La variable dicotómica NIIF es estadísticamente significativa con signo positivo para la muestra de empresas industriales.

e) La variable dicotómica NIIF es estadísticamente significativa con signo negativo para la muestra de empresas financieras.

f) Sorprendentemente, ni el endeudamiento, ni la ROE ni las medidas de cash flow juegan ningún papel, estadísticamente hablando, en explicar el riesgo sistemático de las acciones.

g) Los modelos obtenidos del estudio del periodo 2005-2011 muestran una bondad estadística superior a la de los modelos obtenidos del estudio del periodo 2001-2004. 
h) Los resultados del análisis del periodo 2005-2011 muestran y confirman que 6 variables independientes explican el riesgo de las acciones industriales: el tamaño, el ratio BAIT/VEN, el Euribor, la tasa de desempleo, el TIL y el PIB.

i) Los resultados del análisis del periodo 2005-2011 confirman que 5 indicadores explican el riesgo de las acciones financieras: el tamaño, el ratio BAIT/AT, el Euribor, la tasa de desempleo $\mathrm{y}$ el PIB.

La investigación confirma que existe conexión entre el riesgo y la información contable y macroeconómica, lo que demuestra que no solo la información contable explica el riesgo sistemático de una acción y, en consecuencia, su coste de capital.

El estudio realizado permite dar respuesta a los 2 objetivos inicialmente planteados, pues los resultados proporcionan información útil para tomar decisiones financieras racionales, tanto por los directivos de la empresa como para los profesionales externos, y también queda demostrado que la adopción de las NIIF afecta a la relación, ya que es una variable estadísticamente significativa.

Los resultados conseguidos tienen una directa implicación práctica para el empresario, pues representan un avance en cómo medir o estimar el riesgo cuando la empresa no cotiza en bolsa. El trabajo indica qué variables hay que considerar y observar para estimar el riesgo empresarial y, posteriormente, determinar la rentabilidad mínima exigida o tasa de actualización.

Los resultados nos dicen cómo actuar para que una variable muy importante en el ámbito de las decisiones financieras (el coste de capital) no se fije arbitrariamente y se determine de una forma más racional. El trabajo nos indica qué variables observar para podernos anticipar a un cambio en el riesgo y, por lo tanto, en la tasa de actualización o coste de capital de las acciones y poder desarrollar una gestión más eficiente. Evidentemente, esta investigación tiene las limitaciones propias del tamaño y de las características de la muestra analizada.

Los estudios realizados con datos de otros países (Landsman et al., 2012, Papadamou y Tzivinikos, 2013 y Kim et al., 2014) permiten una cierta comparación pero poca, ya que solo uno de los trabajos (Papadamou y Tzivinikos, 2013) utiliza la misma variable dependiente. Este trabajo utiliza datos de los bancos griegos y muestra que con la aplicación de las NIIF aparecen más variables contables que explican el riesgo sistemático. La investigación concluye que la aplicación de las NIIF afecta al contenido informativo de las variables contables, y esto influye en el riesgo sistemático.

Respecto a los otros 2 trabajos (Landsman et al., 2012, y Kim et al., 2014), el primero analiza los efectos de las NIIF en el contenido informativo del beneficio anual para una muestra de 27 países (16 que aplican las NIIF y 11 que no las aplican), y los resultados revelan que los países que han adoptado las nuevas normas muestran un incremento en la rentabilidad, y el segundo trabajo estudia los efectos de las NIIF en las empresas rusas. Los resultados muestran que el tamaño explica la rentabilidad de las acciones con signo negativo y que la información publicada por las empresas que aplican las NIIF está mejor valorada que la publicada por empresas que todavía no han adoptado las NIIF.

\section{Apéndice 1. Coeficientes de correlación entre las variables independientes}

\begin{tabular}{llllllll}
\hline & $\mathrm{Bi}_{\text {bex35 }}$ & $\mathrm{SOL}$ & $\mathrm{RE}$ & $\mathrm{TA}$ & $\mathrm{CR}$ & $\mathrm{EO}$ & $\mathrm{EF}$ \\
\hline $\mathrm{Bi}_{\text {bex35 }}$ & 1 & 1 & & & & \\
$\mathrm{SOL}$ & -0.0026 & 1 & & & & \\
$\mathrm{RE}$ & 0.0304 & -0.0110 & 1 & & &
\end{tabular}




\begin{tabular}{|c|c|c|c|c|c|c|c|}
\hline TA & $0.2944 *$ & $-0.0696^{*}$ & 0.0422 & 1 & & & \\
\hline $\mathrm{CR}$ & 0.0325 & -0.0214 & 0.0122 & $0.3684^{*}$ & 1 & & \\
\hline $\mathrm{EO}$ & $-0.0857^{*}$ & -0.0066 & 0.0066 & 0.0451 & $0.0817 *$ & 1 & \\
\hline $\mathrm{EF}$ & -0.0378 & 0.0093 & 0.0042 & 0.0023 & $-0.0568^{*}$ & 0.0036 & 1 \\
\hline VAB/VCFP & -0.0171 & 0.0018 & $-0.4728^{*}$ & -0.0056 & 0.0124 & 0.0108 & 0.0022 \\
\hline VAN/VCFP & -0.0178 & 0.0040 & $-0.4864^{*}$ & -0.0107 & 0.0086 & 0.0102 & 0.0009 \\
\hline VAB/VENT & $-0.0988^{*}$ & -0.0073 & 0.0173 & $0.0732 *$ & $0.0997 *$ & $0.9288 *$ & 0.0093 \\
\hline VAN/VENT & $-0.0822 *$ & -0.0060 & 0.0162 & $0.0630 *$ & $0.0855^{*}$ & $0.7630 *$ & 0.0066 \\
\hline VAB/AT & 0.0087 & 0.0373 & 0.0219 & -0.0425 & $0.0556^{*}$ & $0.0583^{*}$ & -0.0043 \\
\hline VAN/AT & -0.0021 & 0.0416 & 0.0245 & -0.0193 & $0.0626^{*}$ & 0.0512 & -0.0077 \\
\hline $\mathrm{VAB} / \mathrm{GF}$ & -0.0054 & $0.9033^{*}$ & -0.0037 & -0.0272 & 0.0095 & 0.0238 & 0.0015 \\
\hline VAN/GF & 0.0030 & $0.9398^{*}$ & -0.0027 & -0.0206 & 0.0085 & 0.0187 & 0.0009 \\
\hline BAIT/AT & 0.0154 & 0.0332 & -0.0089 & -0.0292 & 0.0092 & $0.1441 *$ & 0.0208 \\
\hline BAIT/GF & -0.0043 & $0.8634 *$ & -0.0046 & -0.0269 & 0.0077 & 0.0451 & 0.0027 \\
\hline BAIT/VCFP & 0.0474 & -0.0222 & $-0.1062 *$ & $0.1013 *$ & 0.0220 & 0.0421 & 0.0198 \\
\hline BAIT/VENT & $-0.0856^{*}$ & -0.0066 & 0.0066 & 0.0451 & $0.0808 *$ & $1.0000 *$ & 0.0036 \\
\hline BN/AT & 0.0328 & $0.0592 *$ & -0.0004 & -0.0222 & 0.0055 & $0.1105^{*}$ & -0.0440 \\
\hline $\mathrm{BN} / \mathrm{GF}$ & -0.0004 & $0.8699 *$ & -0.0047 & -0.0255 & 0.0062 & 0.0447 & 0.0094 \\
\hline BN/VCFP & 0.0434 & 0.0022 & 0.0390 & $0.0534 *$ & -0.0141 & 0.0266 & 0.0007 \\
\hline BN/VENT & 0.0524 & -0.0004 & -0.0046 & 0.0419 & 0.0520 & $0.4815^{*}$ & -0.0570 \\
\hline CF1/VCFP & 0.0108 & 0.0019 & 0.0053 & -0.0028 & -0.0187 & -0.0001 & 0.0007 \\
\hline CF2/VCFP & -0.0006 & 0.0017 & 0.0053 & -0.0052 & -0.0264 & -0.0006 & 0.0008 \\
\hline CF3/VCFP & 0.0100 & 0.0019 & 0.0053 & -0.0025 & -0.0186 & -0.0000 & 0.0007 \\
\hline CF1/VENT & $0.0591 *$ & 0.0087 & 0.0015 & -0.0257 & $-0.0828 *$ & $-0.3978^{*}$ & 0.0012 \\
\hline CF2/VENT & $0.0555^{*}$ & 0.0101 & -0.0020 & -0.0333 & $-0.1024 *$ & $-0.5340^{*}$ & 0.0010 \\
\hline CF3/VENT & $0.0590 *$ & 0.0056 & 0.0039 & -0.0140 & -0.0517 & $-0.1930 *$ & 0.0014 \\
\hline CF1/AT & $-0.0691 *$ & 0.0019 & 0.0053 & $0.1049 *$ & $0.1962 *$ & -0.0007 & 0.0008 \\
\hline $\mathrm{CF} 2 / \mathrm{AT}$ & $0.0692 *$ & 0.0016 & 0.0052 & $-0.1070 *$ & $-0.2099 *$ & -0.0032 & 0.0009 \\
\hline CF3/AT & $-0.0691^{*}$ & 0.0019 & 0.0053 & $0.0846^{*}$ & $0.1529 *$ & -0.0002 & 0.0007 \\
\hline $\mathrm{CF} 1 / \mathrm{GF}$ & 0.0107 & 0.0083 & 0.0064 & 0.0016 & -0.0198 & -0.0012 & 0.0010 \\
\hline $\mathrm{CF} 2 / \mathrm{GF}$ & $0.0586^{*}$ & 0.0046 & 0.0064 & 0.0008 & -0.0218 & -0.0084 & 0.0010 \\
\hline $\mathrm{CF} 3 / \mathrm{GF}$ & 0.0134 & 0.0044 & 0.0064 & 0.0016 & -0.0196 & -0.0002 & 0.0010 \\
\hline EUR & $-0.1331 *$ & 0.0036 & -0.0123 & 0.0393 & 0.0534 & -0.0011 & 0.0003 \\
\hline TIL & $0.0768^{*}$ & -0.0138 & -0.0357 & 0.0450 & $-0.1015^{*}$ & -0.0029 & 0.0420 \\
\hline IPC & $0.0931 *$ & -0.0211 & 0.0052 & 0.0229 & 0.0124 & -0.0125 & 0.0078 \\
\hline TD & $-0.2526^{*}$ & -0.0418 & -0.0108 & $-0.1368 *$ & $-0.0697 *$ & 0.0089 & -0.0101 \\
\hline PIB & $-0.2656^{*}$ & -0.0209 & 0.0062 & $-0.1968 *$ & 0.0416 & -0.0039 & -0.0463 \\
\hline DJ & 0.0352 & 0.0322 & -0.0004 & -0.0082 & $0.0796 *$ & -0.0165 & -0.0208 \\
\hline S\&P500 & -0.0096 & 0.0241 & -0.0106 & -0.0213 & $0.0777 *$ & -0.0146 & -0.0296 \\
\hline
\end{tabular}

VAN/GF BAIT/AT BAIT/GF BAIT/VCFP BAIT/VENT BN/AT $\quad$ BN/GF

\begin{tabular}{llllllll}
\hline VAN/GF & 1 & & & & \\
BAIT/AT & 0.0518 & 1 & & & & \\
BAIT/GF & $0.9516^{*}$ & $0.0644^{*}$ & 1 & & & \\
BAIT/VCFP & 0.0071 & $0.5263^{*}$ & 0.0047 & 1 & & \\
BAIT/VENT & 0.0187 & $0.1440^{*}$ & 0.0451 & 0.0421 & 1 & & \\
BN/AT & 0.0491 & $0.6140^{*}$ & $0.0601^{*}$ & $0.2408^{*}$ & $0.1105^{*}$ & 1 & \\
BN/GF & $0.9545^{*}$ & 0.0538 & $0.9954^{*}$ & -0.0020 & 0.0447 & $0.0723^{*}$ & 1 \\
BN/VCFP & 0.0103 & $0.2447^{*}$ & 0.0091 & $0.4542^{*}$ & 0.0266 & $0.4649^{*}$ & 0.0134 \\
BN/VENT & 0.0105 & 0.0375 & 0.0271 & -0.0275 & $0.4815^{*}$ & $0.2394^{*}$ & $0.0720^{*}$ \\
CF1/VCFP & -0.0008 & -0.0077 & -0.0014 & -0.0022 & -0.0001 & -0.0073 & -0.0016 \\
CF2/VCFP & -0.0008 & -0.0071 & -0.0014 & -0.0018 & -0.0006 & -0.0066 & -0.0016 \\
CF3/VCFP & $-0.0008-$ & 0.0076 & -0.0014 & -0.0023 & -0.0000 & -0.0072 & -0.0016 \\
CF1/VENT & -0.0018 & -0.0230 & -0.0029 & -0.0155 & $-0.3978^{*}$ & -0.0143 & -0.0025 \\
CF2/VENT & -0.0019 & -0.0248 & -0.0029 & -0.0185 & $-0.340 *$ & -0.0136 & -0.0023
\end{tabular}




\begin{tabular}{llllllll} 
CF3/VENT & -0.0014 & -0.0160 & -0.0022 & -0.0092 & $-0.1930^{*}$ & -0.0108 & -0.0021 \\
CF1/AT & -0.0008 & 0.0125 & -0.0014 & -0.0023 & -0.0007 & 0.0074 & -0.0016 \\
CF2/AT & -0.0009 & -0.0166 & -0.0015 & -0.0017 & -0.0031 & -0.0114 & -0.0017 \\
CF3/AT & -0.0008 & 0.0072 & -0.0014 & -0.0023 & -0.0002 & 0.0033 & -0.0016 \\
CF1/GF & 0.0070 & -0.0096 & 0.0070 & -0.0029 & -0.0012 & -0.0082 & 0.0068 \\
CF2/GF & 0.0019 & -0.0103 & 0.0015 & -0.0033 & -0.0084 & -0.0084 & 0.0014 \\
CF3/GF & 0.0019 & -0.0098 & 0.0015 & -0.0029 & -0.0002 & -0.0084 & 0.0013 \\
EUR & -0.0140 & -0.0440 & -0.0033 & -0.0379 & -0.0011 & -0.0283 & -0.0057 \\
TIL & -0.0240 & $0.0757^{*}$ & -0.0254 & $0.0722^{*}$ & -0.0028 & $0.0776^{*}$ & -0.0276 \\
IPC & -0.0286 & $-0.1387^{*}$ & -0.0089 & $-0.1306^{*}$ & -0.0124 & $-0.1177^{*}$ & -0.0092 \\
TD & -0.0234 & -0.0196 & -0.0295 & 0.0077 & 0.0088 & -0.0104 & -0.0327 \\
PIB & -0.0036 & $-0.0932^{*}$ & -0.0136 & $-0.0782^{*}$ & -0.0040 & $-0.1302^{*}$ & -0.0160 \\
DJ & 0.0318 & -0.0461 & 0.0227 & -0.0392 & -0.0165 & $-0.0702^{*}$ & 0.0249 \\
S\&P500 & 0.0202 & $-0.0553^{*}$ & 0.0082 & -0.0422 & -0.0146 & $-0.0868^{*}$ & 0.0094 \\
& & & & & & & \\
& EUR & TIL & IPC & TD & PIB & DJ & S\&P500 \\
\hline EUR & 1 & & & & & \\
TIL & $-0.1991^{*}$ & 1 & & & & & \\
IPC & $0.1676^{*}$ & $-0.1906^{*}$ & 1 & & & & \\
TD & $-0.1585^{*}$ & $0.1854^{*}$ & $-0.4770^{*}$ & 1 & & & \\
PIB & $-0.2273^{*}$ & $-0.3979^{*}$ & -0.0493 & $0.3866^{*}$ & 1 & & \\
DJ & $-0.1074^{*}$ & $-0.6436^{*}$ & $0.2290^{*}-$ & $-0.5036^{*}$ & $0.3278^{*}$ & 1 & \\
S\&P500 & -0.0088 & $-0.6024^{*}$ & $0.2360^{*}$ & $-0.4622^{*}$ & $0.3709^{*}$ & $0.9700^{*}$ & 1 \\
\hline
\end{tabular}

\section{Referencias}

Agusman, A., Monroe, G. S., Gasbarro, D. y Zumwalt, J. K. (2008). Accounting and capital market measures of risk: Evidence from Asian banks during 1998-2003. Journal of Banking \& Finance, 32, 480-488.

Arfaoui, M. y Abaoub, E. (2010). The determinants of systematic risks: International evidence from the macro-finance interface. Journal of Advanced Studies in Finance, 2, 119-141. I.

Baltagi, B. H. (2012). Econometric Analysis of Panel Data (4ª edición). Wiley.

Beaver, W., Kettler, P. y Scholes, M. (1970). The association between market-determined and accounting-determined risk measures. The Accounting Review, October, 655-682.

Beck, N. y Katz, J. (2001). Time-series cross-section data: What have we learned in the past few years? Annual Review of Political Science, 4, 271-293.

Bildersee, J. S. (1975). The association between a market-determined measures of risk and alternative measures of risk. The Accounting Review, 50(1), 81-98.

Brealey, R. A., Myers, S. C. y Allen, F. (2010). Principios de Finanzas Corporativas (9ª edición). McGraw-Hill.

Breen, W. J. y Lerner, E. M. (1973). Corporate financial strategies and market measures of risk and return. The Journal of Finance, 28(2), 339-351.

Brimble, M. y Hodgson, A. (2007). Assessing the risk relevance of accounting variables in diverse economic conditions. Managerial Finance, 33(8), 553-573.

Campbell, J. Y., Polk, C. y Vuolteenaho, T. (2010). Growth or glamour' fundamentals and systematic risk in stock returns. The Review of Financial Studies, 23, 305-334.

Chen, M.-H. (2013). Risk determinants of China's hotel industry. Tourism Economics, 19, 77-99.

Christensen, H. B., Hail, L. y Leuz, C. (2013). Mandatory IFRS reporting and changes in enforcement. Journal of Accounting and Economics, 56, 147-177.

Da, Z. y Warachka, M. C. (2009). Casflow risk systematic earnings revisions, and the cross-section of stock returns. Journal of Financial Economics, 94, 448-468.

Damodaran, A. (2012). Applied Corporate Finance ( $3^{\text {a }}$ edición). John Wiley \& Sons.

Daske, H., Hail, L., Leuz, C. y Verdi, R. (2013). Adopting a label: Heterogeneity in the economic consequences around IAS/IFRS adoptions. Journal of Accounting Research, 51(3), 495-547. 
Elgers, P. T. y Murray, D. (1982). The impact of the choice of market index on the empirical evaluation of accounting risk measures. The Accounting Review, 57(2), 358-375.

Elmoatasem, K. (2005). Disclosure of market risk or accounting measures of risk: An empirical study. Managerial Auditing Journal, 20(8), 867-875.

Elyasiani, E. y Mansur, I. (2005). The association between market and exchange rate risks and accounting variables: AGARCH model of the Japanese banking institutions. Review of Quantitative Finance and Accounting, 25, $183-206$.

Foster, G., Kasnik, R. y Sidhu, B. K. (2012). International equity variation: the relative importance of country and industry factors versus company-specific financial reporting information. Accounting \& Finance, 52(3), 767-814.

Greene, W. H. (2012). Econometric Analysis (7 $7^{\mathrm{a}}$ edición). Prentice Hall.

Gosnell, T. y Nejadmalayeri, A. (2010). Macroeconomic news and risk factor innovations. Managerial Finance, 36(2), 566-582.

Hamao, Y., Masulis, R. W. y Ng, V. (1990). Correlation in price changes and volatility across international stock markets. Review of Financial Studies, 3(2), 281-307.

Han, F. y He, H. (2013). Cost of equity capital of foreign firms. Review of Accounting and Finance, 12(3), 268-285.

Hsiao, C. (2003). Analysis of Panel Data. Cambridge University Press.

Ismail, B. y Kim, M. (1989). On the association of cash flow variables with market risk: Further evidence. The Accounting Review, 64(1), 125-136.

Karpik, P. y Belkaoui, A. (1990). The relative relationship between systematic risk and value added variables. Journal of International Financial Management and Accounting, 1(3), 259-275.

Kim, J.-B., Shi, H. y Zhou, J. (2014). International Financial Reporting Standards, institutional infrastructures, and implied cost of equity capital around the world. Review of Quantitative Finance and Accounting, 42, 469-507.

Landsman, W. R., Maydew, E. L. y Thornock, J. R. (2012). The information content of annual earnings announcements and mandatory adoption of IFRS. Journal of Accounting and Economics, 53, 34-54.

Lee, J.-S. y Jang, S. (2007). The systematic-risk determinants of the US airline industry. Tourism Management, 28(2), $434-442$.

Lev, B. (1974). On the association between operating leverage and risk. Journal of Financial and Quantitative Analysis, September, 627-641.

Lev, B. y Kunitzky, S. (1974). On the association between smoothing measures and the risk of common stocks. Accounting Review, 49(2), 259-271.

Li, S. (2010). Does mandatory adoption of international financial reporting standards in the European Union reduce the cost of equity capital? The Accounting Review, 85(2), 607-636.

Lord, R. A. (1996). The impact of operating and financial risk on equity risk. Journal of Economics and Finance, 20(3), 27-38.

Menéndez, C., Prior, D. y Orgaz, N. (2012). ¿Existe relación entre la información contable y el riesgo sistemático de las empresas? Academia Revista Latinoamericana de Administración, 49, 46-60.

Papadamou, S. y Tzivinikos, T. (2013). The risk relevance of international financial reporting standards: evidence from greek banks. International Review of Financial Analysis, 27, 43-54.

Pulido, A. y Pérez, J. (2001). Modelos Econométricos. Ediciones Pirámide.

Sloan, R. G. (1996). Do stock prices fully reflect information in accruals and cash flows about futures earnings? The Accounting Review, 71(3), 289-315.

Vassalou, M. (2003). News related to future GDP growth as a risk factor in equity returns. Journal of Financial Economics, $68,47-73$

Wooldridge, J. M. (2002). Econometric Analysis of Cross Section and Panel Data. Cambridge, MA: MIT Press. 